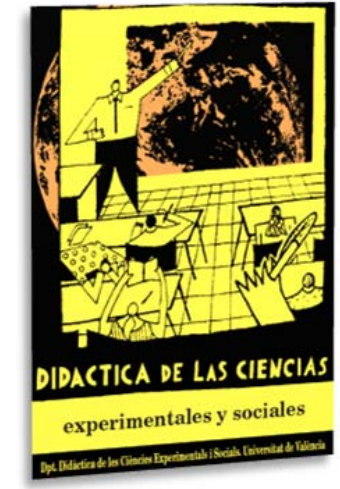

\title{
Análisis de las experiencias de aprendizaje de las ciencias de los/as maestros/as de Educación Infantil y Primaria en formación inicial a través de sus relatos de vida
}

\author{
Analysis of science learning experiences of \\ students of Preschool and Primary school initial \\ teacher training through their life-stories
}

DOI: $10.7203 / D C E S .37 .14303$

Marcia Eugenio-Gozalbo

Universidad de Valladolid, marcia.eugenio@uva.es ORCID iD: http://orcid.org/0000-0002-7907-9780

Juan José Vicente Martorell

Universidad de Cádiz, juanjose.vicente@uca.es

ORCID iD: http://orcid.org/0000-0001-7498-0214

\author{
Lourdes Aragón Núñez \\ Universidad de Cádiz, lourdes.aragon@uca.es \\ ORCID iD: http://orcid.org/0000-0003-3062-9734 \\ Natalia Jiménez-Tenorio \\ Universidad de Cádiz, natalia.jimenez@uca.es \\ ORCID iD: http://orcid.org/0000-0001-7879-9877
}

\begin{abstract}
RESUMEN: Se presenta una investigación sobre las experiencias de aprendizaje de las ciencias de los maestros/as en formación inicial de dos universidades españolas. Se recogieron 263 relatos de vida entre estudiantes de Grado en Educación Infantil y Primaria -a partes iguales en que narraban sus vivencias en las etapas de Infantil, Primaria y Secundaria, y también en entornos no académicos. Estos relatos se analizaron cualitativamente, mediante la definición de categorías y subcategorías de contenido, y cuantitativamente, mediante el recuento de la frecuencia de aparición de fragmentos clasificados en ellas. Se presentan resultados respecto a contenidos, recursos, aproximaciones metodológicas, aspectos subjetivos y aprendizaje en entornos no académicos. Estos evidencian la necesidad de promover, desde la formación inicial de maestros/as, experiencias de aprendizaje de las ciencias enmarcadas en un modelo constructivista, que impliquen un papel protagonista del estudiante, el uso de metodologías activas y de contextos de aprendizaje distintos al aula.
\end{abstract}

Palabras Clave: enseñanza-aprendizaje de las ciencias, formación inicial de maestros/as, relatos de vida.

Abstract: We present a research on science learning experiences of initial teacher training students at two Spanish universities. We collected 263 life-stories among students of Degree in Preschool and Primary Education -equal halves-, where students explained their experiences at Preschool, Primary and Secondary Education, and at non-academic contexts. These life-stories were qualitatively analyzed, by defining content categories and subcategories, and also quantitatively analyzed, by counting the frequency of appearance of fragments classified within them. We present results regarding contents, didactic resources, methodological approaches, subjective aspects, and learning at non-academic contexts. These clearly show the need to promote, from initial teacher training, science learning experiences framed in a constructivist model involving a leading role of students, and the use of active methodologies and learning contexts other than the classroom.

KEYWORDs: science teaching and learning, initial teacher training, life-stories

Fecha de recepción: mayo de 2019 Fecha de aceptación: octubre de 2019

Este trabajo se ha realizado en el marco del Proyecto de Innovación Docente "La alfabetización científica-tecnológica en la formación inicial de maestros/as: saber de ciencias, saber hacer ciencias, saber acerca de las ciencias y actuar desde la ciencia”, concedido en la convocatoria de Proyectos de Innovación y Mejora Docente de la Universidad de Cádiz (UCA) durante el curso académico 2015/2016. 


\section{INTRODUCCIÓN}

La construcción de un modelo educativo que desde edades tempranas contribuya a la formación integral de la persona, el desarrollo de su pensamiento crítico y científico, su calidad de vida y su participación social como ciudadano/a responsable es considerado una prioridad (Tedesco, 2006). El papel del docente y su profesionalidad son centrales, no sólo en relación a los procesos de enseñanza-aprendizaje, sino también a su compromiso con la educación, los contextos educativos, las familias, el entorno y el conocimiento, porque el profesorado es el factor que verdaderamente determina el éxito o el fracaso de cualquier reforma o innovación curricular (Tobin, Tippins y Gallard, 1994). Cuando los docentes enseñan ciencias, ponen en juego conocimientos, concepciones y actitudes sobre ellas y sobre la forma de aprenderlas y enseñarlas, que son el resultado de sus años de trayectoria académica, de su formación profesional específica, y de su práctica cotidiana en la actividad docente (Ferreyra, 2012). La identidad personal y profesional de un docente implica, por tanto, considerar tres dominios que se integran de manera simultánea y que configuran la actividad profesional: dominio conceptual, emocional-afectivo y práctico (Pontes, Serrano y Poyatos, 2013).

Los estudios acerca del pensamiento del profesor tienen una larga trayectoria en la investigación en didáctica, y analizan las interpretaciones, los significados y los conocimientos que subyacen a la práctica docente. Muchos de ellos coinciden en la relevancia que revisten las ideas, concepciones y actitudes del profesorado sobre la ciencia y sobre la forma de aprenderla y enseñarla para su práctica cotidiana (Porlán y Rivero, 1998). Estas concepciones son a menudo implícitas, y resultan más estables cuanto más tiempo han perdurado. Se ha visto que las concepciones pedagógicas personales, adquiridas de forma natural y no reflexiva a partir de las propias experiencias escolares, suponen un obstáculo para la formación y el cambio didáctico del profesorado (Carniatto y Fossa, 1998). Como señala Delval (2002): “cambiar los profesores es algo extremadamente difícil (...) Enseñan, sobre todo, como les enseñaron a ellos (...)”. O, en palabras de Gil-Pérez (1991):

Los profesores tienen ideas, actitudes y comportamientos sobre la enseñanza debidos a una larga formación 'ambiental' durante el periodo en que fueron alumnos. La influencia de esta formación incidental es enorme porque responde a experiencias reiteradas y se adquiere de forma no reflexiva como algo natural, obvio, 'de sentido común', escapando así a la crítica y convirtiéndose en un verdadero obstáculo.

Además, en muchas ocasiones tales concepciones están alejadas de los puntos de vista que sostienen la filosofía y sociología de la ciencia en la actualidad, y de los modelos didácticos innovadores (Porlán, Rivero y Martín, 1997, 1998).

Así, cuando los futuros maestros/as comienzan su etapa universitaria, lo hacen con un bagaje de conocimientos, valores, creencias, roles y actitudes sobre la ciencia, la enseñanza de las ciencias, el profesor, etc. que son fruto de su trayectoria académica anterior. En este estudio empírico abordamos las vivencias de aprendizaje de las ciencias de estudiantes de los Grados en Educación Infantil y Primaria de dos universidades españolas, la Universidad de Cádiz (UCA), y la Universidad de Valladolid - Campus de Soria (UVa). Lo hacemos en base a sus propios relatos, aproximándonos mediante una técnica cualitativa, la investigación biográfico-narrativa, que se basa en el análisis los relatos personales de historias de vida, para saber de qué modo han valorado e integrado dichas vivencias. Partir de ellas es relevante, porque así se proporciona oportunidades a los estudiantes de ser conscientes de sus concepciones, actitudes y prácticas, de modo que puedan reestructurarlas e ir desarrollando su modelo didáctico personal, y además continuar formándose autónomamente (Sanmartí, 2001). 


\section{MARCO TEÓRICO}

Lederman (1992) realizó una exhaustiva revisión de los estudios sobre concepciones o creencias para el periodo 1954-1992, identificando en ellos cuatro etapas consecutivas: la primera, caracterizada por abordar las ideas de los estudiantes; la segunda, por introducir la dimensión curricular; la tercera, por considerar las concepciones de los docentes; y la cuarta, por estudiar las relaciones entre el pensamiento del docente y sus prácticas de enseñanza. Inicialmente se aceptó una relación directa y unívoca entre las concepciones del docente y su acción en el aula. Sin embargo, tras detectarse desfases e inconsistencias entre ambas (Lederman, 1992; Mellado, 1996), se postuló que los conocimientos académicos o teórico-proposicionales no se transfieren directamente a la práctica (Bryan y Abell, 1999), y que el docente requiere de conocimientos procedimentales y esquemas prácticos de acción en el aula para que esa transferencia sea posible (Furió y Carnicer, 2002; Tobin, 1993).

Más recientemente, han sido objeto de atención -por su importancia para la mejora cualitativa de los sistemas educativos- la evolución de las concepciones (Ruiz, Da Silva, Porlán y Mellado, 2005) y el cambio didáctico (Mellado, 2001, 2003) del profesorado de ciencias experimentales. Algunos programas de formación de base constructivista señalan que, para promover tales cambios, es necesario incluir los valores y las experiencias de los sujetos (Furió y Carnicer, 2002; Sanmartí, 2001). Para ello previamente sería preciso explicitarlos previamente, tal y como pretende este trabajo, y señalan investigaciones recientes en el campo de la didáctica de las ciencias, que evidencian que explicitar los modelos mentales y reflexionar sobre las concepciones del alumnado parece contribuir significativamente al conocimiento didáctico del contenido (Vázquez-Alonso y Manassero-Mas, 2015).

En relación a las experiencias, nos planteamos el componente afectivo como parte relevante de los aprendizajes. Se ha señalado que el cambio conceptual es tanto cognitivo como afectivo (Thagard, 2008), por lo que el profesorado que ignora los aspectos afectivos del aprendizaje puede estar limitando el cambio conceptual de su alumnado (Mellado, Borrachero, Brígido, Melo, Dávila, Cañada, Conde, Costillo, Cubero, Esteban, Martínez, Ruiz y Sánchez, 2014). En este sentido, existen ya propuestas estratégicas para la puesta en práctica de programas de educación emocional para el profesorado, con el objetivo de que éste, a su vez, pueda introducir elementos de educación emocional en su práctica docente (Bisquerra y Pérez, 2012; Muñoz y Bisquerra, 2013). Como indican los estudios de Olitsky y Milne (2012) y Vázquez y Manassero (2007), los estados emocionales positivos favorecen el aprendizaje y el compromiso de los estudiantes como aprendices activos, mientras que los negativos limitan su capacidad de aprender. En los últimos años, algunos autores apuntan que las emociones sobre el proceso de enseñanza-aprendizaje de cada materia deben considerarse parte del conocimiento didáctico del contenido (Garritz, 2010; Garritz y Ortega-Villar, 2013), entendido como una forma de razonamiento y acción didáctica por la cual el docente transforma un contenido en representaciones comprensibles y vivenciales para sus estudiantes.

\section{OBjetivos}

Las asignaturas específicamente relacionadas con las ciencias experimentales y con su enseñanza-aprendizaje son, en el caso de la UCA, una única asignatura de carácter obligatorio denominada Didáctica del Medio Natural en el Grado en Educación Infantil (GEI, de ahora en adelante), y dos asignaturas obligatorias denominadas Didáctica de las Ciencias de la Naturaleza 1 (CN1) y Didáctica de las Ciencias de la Naturaleza 2 (CN2) en el Grado en Educación Primaria (GEP, de ahora en adelante), ambas cuatrimestrales y de 6 ECTS. Para el caso de la UVa-Soria, una única asignatura de carácter obligatorio, cuatrimestral y de 9 ECTS, denominada Las Ciencias de la Naturaleza en el currículo de la Educación Infantil en el GEI. 
En este trabajo se presenta una investigación realizada entre los estudiantes de estas cuatro asignaturas en el curso 2015/2016, cuyo objetivo fundamental fue indagar acerca de las experiencias que han vivido en relación a la enseñanza-aprendizaje de las ciencias a lo largo de las etapas educativas previas a su formación universitaria, incluyendo Infantil, Primaria, Secundaria y Bachillerato o FP, y también entornos no formales, para comprender mejor las potenciales causas sistémicas de la desmotivación con que abordan las asignaturas de ciencias en su formación inicial de maestros/as.

Cabe aquí señalar que el colectivo objeto de este estudio es muy relevante, en tanto en cuanto será el responsable de las primeras aproximaciones de los niños y niñas hacia los conocimientos y procedimientos de las ciencias, y contribuirá al desarrollo de sus actitudes hacia las ciencias y hacia su aprendizaje desde edades tempranas y a lo largo de la Educación Primaria.

\section{Metodología}

La recogida de datos se llevó a cabo durante el curso académico 2015/2016, entre estudiantes de segundo y tercer curso de GEI y GEP de la Facultad de Ciencias de la Educación de la UCA y de tercer curso de GEI de la Facultad de Educación de Soria (UVa). Inicialmente, se llevó a cabo una caracterización sociométrica de los participantes, en base a un cuestionario de elaboración propia que se administró mediante Google Forms, y en que se les pedía información sobre su edad, sexo, estudios anteriores, Comunidad Autónoma de procedencia, y elección de asignaturas en los estudios precedentes (Secundaria y Bachillerato o FP), entre otros.

Para la investigación se utilizó un procedimiento enmarcado en un enfoque cualitativo de investigación, una denominación paraguas que incluye una diversidad de marcos de interpretación, como la fenomenología, el naturalismo, el interpretativismo, etc. (Savin-Baden y Major, 2013; Sparkes y Smith, 2014). En el enfoque cualitativo la recogida de datos se centra en obtener las perspectivas y puntos de vista de los participantes (sus emociones, prioridades, experiencia y significados), y su lógica es más bien inductiva, pues se explora y describe la realidad para luego generar perspectivas teóricas (Miles, Huberman y Saldaña, 2014). En este estudio se empleó para la recogida de datos una técnica biográfico-narrativa. Las técnicas narrativas comprenden por una parte las autobiografías o relatos de vida, y por otra las biografías o historias de vida; entre ambas la diferencia básica es el narrador: el propio protagonista, en el primer caso, y el investigador, en el segundo. Según Tójar (2006):

En las biografías, los motivos, los porqués de determinados acontecimientos y trayectorias poseen un gran interés, para determinar las causas y las consecuencias acontecidas a lo largo de una vida (Tójar, 2006, p. 260).

Es por ese motivo que se escogió esta aproximación metodológica, pues el objetivo de nuestra investigación es explicar, sobre la base de las experiencias de enseñanza-aprendizaje de las ciencias previas de nuestro alumnado, la desmotivación o el miedo hacia las disciplinas científicas que apreciamos en él. Según Tójar (2006), la autobiografía o relato de vida:

puede ser más o menos amplia, o referirse a etapas o aspectos concretos de la vida del protagonista, y puede haberse originado por iniciativa propia o a requerimiento de terceros" (Tójar, 2006, p. 258)

Así, al comienzo de las asignaturas se solicitó a los estudiantes que narraran por escrito su experiencia vivida y sentida en relación a la enseñanza-aprendizaje de las ciencias a lo largo de su trayectoria académica, diferenciando las etapas de Infantil, Primaria, Secundaria y los contextos no formales. El ejercicio fue de elaboración libre en cuento a longitud y nivel de detalle, y lo llevaron a 
cabo un total de 263 estudiantes. Se recogió en formato word o pdf, es decir, escrito a ordenador (Imagen 1).

IMAGEN 1. Extracto de una de las narraciones autobiográficas analizadas (Individuo 18)

\title{
RELATO AUTOBIOGRÁFICO: MIS EXPERIENCIAS SENTIDAS DE APRENDIZAJE DE LAS CIENCIAS
}

\begin{abstract}
Mi recuerdo en infantil a cerca de las ciencias es muy limitado, supongo que esto es debido a mi corta edad por aquel entonces. Por una parte, recuerdo que tratábamos mucho el tema de las estaciones y los principales cambios que se producían en cada una de ellas. En invierno por ejemplo, me acuerdo que a través de la nieve tratábamos el tema del agua en sus distintos estados ; en primavera tratábamos el tema de la flora y de cómo crecían las plantas, de hecho plantamos en un bote de yogurt lentejas para seguir el proceso de crecimiento; en verano aprovechando el buen tiempo recuerdo que nos llevaron a una granja de vacas que hay en mi pueblo, Arnedo, y nos enseñaron cómo era el cuidado de estas y cómo se realizaba el proceso de recogida de la leche, además nos enseñaron el sitio donde tenían a los terneros y cómo les daban biberón con una botella gigante; en otoño nos llevaban al parque a recoger hojas y nos explicaban los diferentes frutos secos característicos de esta época.
\end{abstract}

Después, una vez ya en primaria me acuerdo que en los temas relacionados con los animales era con los que más disfrutaba, eran temas que me causaban una tremenda curiosidad $y$ disfrutaba con todas las explicaciones, ya que desde muy pequeña he estado en contacto con animales y son mi gran pasión. Durante este periodo educativo realizamos una gran de actívidades de salida al campo como por ejemplo: visita a la depuradora, visita a una planta de reciclado, visita al parque de Sierra Cebollera, visita al Parque de los patos y al río Cidacos...

Fuente: elaboración propia

Para la obtención de la información, se llevó a cabo un análisis de contenido manual, es decir, sin apoyo de software especializado. Se siguió un sistema de codificación por inferencia, definiendo las categorías y subcategorías de contenido en base a la información proporcionada por los propios textos analizados. La consideración de categorías y subcategorías no establecidas a priori por el investigador es característica de los estudios enmarcados en un enfoque cualitativo de investigación, en que la acción indagatoria se mueve de manera dinámica en ambos sentidos entre los hechos y su interpretación, resultando un proceso más bien “circular” (Hernández, Fernández y Baptista, 2014).

Inicialmente se seleccionaron de forma aleatoria 20 relatos, que se distribuyeron entre los 4 investigadores para llevar a cabo una lectura en profundidad que permitiese identificar las cuestiones que el alumnado reflejaba en relación a las etapas y entornos anteriormente mencionados. Posteriormente, se llevó a cabo una puesta en común que sirvió para identificar y definir categorías y subcategorías de análisis iniciales. Después se analizaron todo el resto de relatos en base a esas categorías y subcategorías, y añadiendo otras nuevas cuando los datos (la información obtenida de los textos) lo hicieron necesario. Los investigadores mantuvieron varias reuniones para contrastar y consensuar las categorías y subcategorías que aparecieron por inferencia. Del total inicial de 263, siete relatos se descartaron, quedando 256.

Para dotar a esta investigación de una aproximación cuantitativa descriptiva se tuvo en cuento qué número de fragmentos de texto se clasificaron en cada una de las categorías y subcategorías de análisis. Es decir, se determinó, a modo de indicador cuantitativo, la frecuencia con que los estudiantes hicieron referencia a determinados aspectos; estos datos se aportan también como resultados, expresados en porcentaje. 


\section{RESULTADOS}

Los datos de caracterización sociométrica se recogen en las Tabla 1 (población total), Tabla 2 (GEI) y Tabla 3 (GEP), respectivamente. Participaron un total de 263 estudiantes, en su mayoría mujeres (77,9\% frente a 22,1\% de hombres), con edades comprendidas entre 22 y 58 años y 23 años de media. El 22,4\% de los participantes cursaban sus estudios en la Facultad de Educación de Soria (UVa), y el 77,6\% en la Facultad de Ciencias de la Educación de la UCA. Sobre el total, aproximadamente la mitad (el 47,1\%) estudiaba el GEP, y la otra mitad (el 52,9\%) el GEI. En el caso de los estudios de ingreso a la universidad, más de tres cuartas partes de los estudiantes procedían de Bachillerato, mayoritariamente en la modalidad de Humanidades y Ciencias Sociales (72,6\%). Así, la mayoría de los/as alumnos/as (75,5\%) no había cursado ninguna asignatura de ciencias desde hacía 4 años o más, llegando a ser el 85,6\% en el caso de los estudiantes del GEI.

TABLA 1. Caracterización sociométrica de los/as alumnos/as que participaron en el estudio ( $\mathrm{N}=263)$

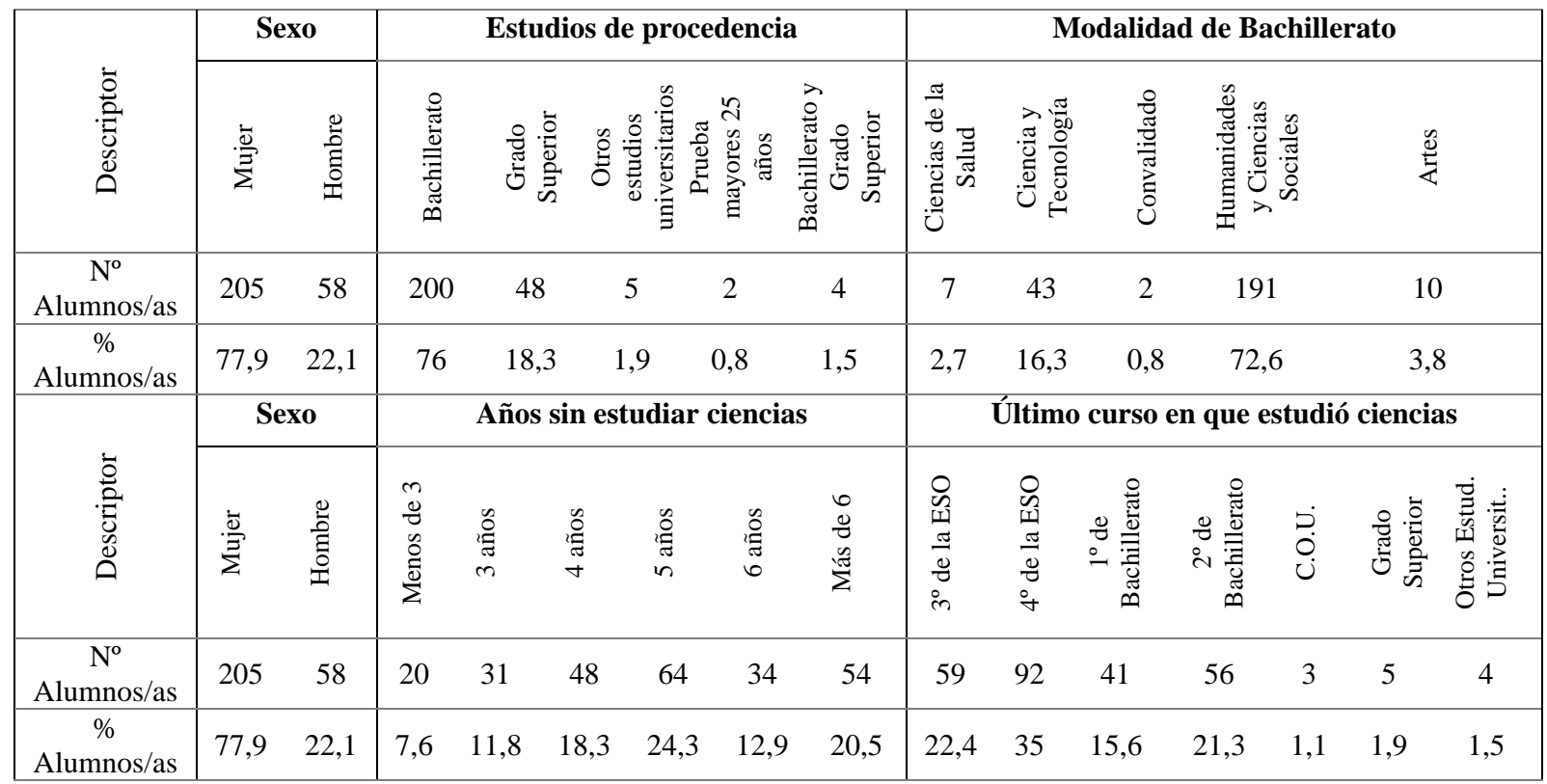

Fuente: elaboración propia 
TABLA 2. Caracterización sociométrica de los/as alumnos/as del GEP que participaron en el estudio ( $\mathrm{N}=124)$

\begin{tabular}{|c|c|c|c|c|c|c|c|c|c|c|c|c|c|c|c|}
\hline \multirow[b]{2}{*}{ 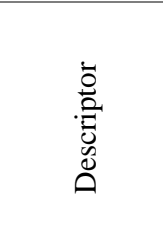 } & \multicolumn{2}{|c|}{ Sexo } & \multicolumn{6}{|c|}{ Estudios de procedencia } & \multicolumn{7}{|c|}{ Modalidad de Bachillerato } \\
\hline & $\stackrel{\frac{\mathscr{g}}{\vec{\Xi}}}{\Sigma}$ & 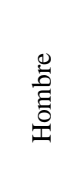 & 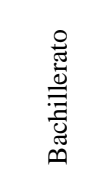 & 覀 & 离 & 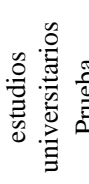 & 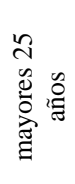 & 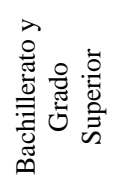 & 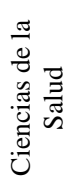 & 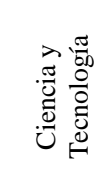 & 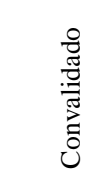 & 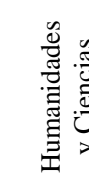 & & 䓠 & \\
\hline $\begin{array}{c}\mathrm{N}^{\circ} \\
\text { Alumnos/as }\end{array}$ & 75 & 49 & 102 & 1 & & 1 & 2 & 4 & 1 & 24 & 0 & 91 & & 6 & \\
\hline $\begin{array}{c}\% \\
\text { Alumnos/as }\end{array}$ & 60,5 & 39, & 82,3 & 11 & 3 & 0,8 & 1,6 & 3,2 & 0,8 & 19,4 & 0 & 73 & & 4,8 & \\
\hline \multirow[b]{2}{*}{ 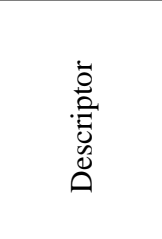 } & \multicolumn{2}{|c|}{ Sexo } & \multicolumn{6}{|c|}{ Años sin estudiar ciencias } & \multicolumn{7}{|c|}{ Último curso en que estudió ciencias } \\
\hline & & $\begin{array}{l}\text { 总 } \\
\text { 总 }\end{array}$ & 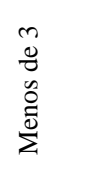 & 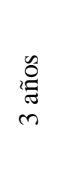 & 总 & 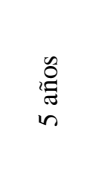 & 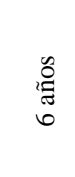 & $\begin{array}{l}0 \\
8 \\
0 \\
: \pi \\
\Sigma\end{array}$ & 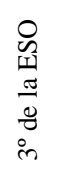 & 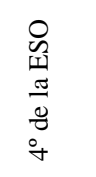 & 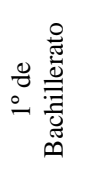 & 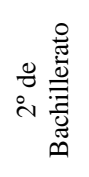 & نُّ & 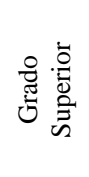 & 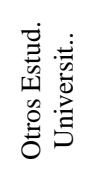 \\
\hline $\begin{array}{c}\mathrm{N}^{\circ} \\
\text { Alumnos/as }\end{array}$ & 75 & 49 & 13 & 18 & 29 & 36 & 15 & 13 & 20 & 43 & 25 & 29 & 0 & 5 & 1 \\
\hline $\begin{array}{c}\% \\
\text { Alumnos/as }\end{array}$ & 60,5 & 39,5 & 10,5 & 14,5 & 23,4 & 29 & 12,1 & 10,5 & 16,1 & 34,7 & 20,2 & 23,4 & 0 & 4 & 0,8 \\
\hline
\end{tabular}

Fuente: elaboración propia

TABLA 3. Caracterización sociométrica de los/as alumnos/as del GEI que participaron en el estudio (N=139)

\begin{tabular}{|c|c|c|c|c|c|c|c|c|c|c|c|c|c|c|c|}
\hline \multirow[b]{2}{*}{ 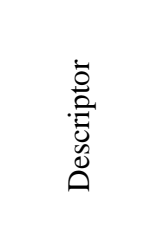 } & \multicolumn{2}{|c|}{ Sexo } & \multicolumn{6}{|c|}{ Estudios de procedencia } & \multicolumn{7}{|c|}{ Modalidad de Bachillerato } \\
\hline & $\stackrel{\mathscr{m}}{\bar{z}}$ & 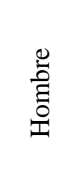 & 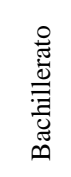 & & 离 & 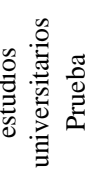 & 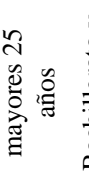 & 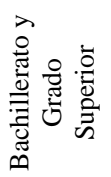 & 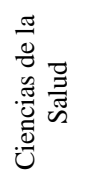 & 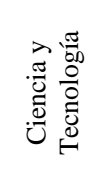 & $\begin{array}{l}\frac{8}{\pi} \\
: \frac{\pi}{7} \\
0 \\
0 \\
0\end{array}$ & 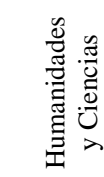 & & 峁 & \\
\hline $\begin{array}{c}\mathrm{N}^{\circ} \\
\text { Alumnos/as }\end{array}$ & 130 & 9 & 98 & 3 & & 4 & 0 & 0 & 6 & 19 & 2 & 100 & & 4 & \\
\hline $\begin{array}{c}\% \\
\text { Alumnos/as }\end{array}$ & 93, & 6,5 & 70,5 & 24 & & 9 & 0 & 0 & 4,3 & 13,7 & 1,4 & 71, & & 2, & \\
\hline \multirow[b]{2}{*}{ 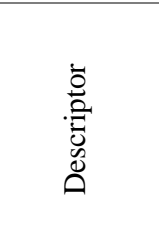 } & \multicolumn{2}{|c|}{ Sexo } & \multicolumn{6}{|c|}{ Años sin estudiar ciencias } & \multicolumn{7}{|c|}{ Último curso en que estudió ciencias } \\
\hline & & 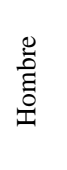 & 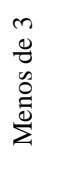 & 总 & 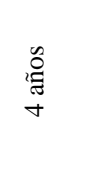 & 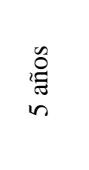 & 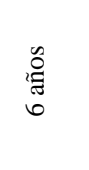 & 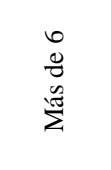 & $\begin{array}{l}0 \\
0 \\
\text { II } \\
\pi \\
\mathbb{0} \\
0 \\
0\end{array}$ & $\begin{array}{l}0 \\
0 \\
\text { In } \\
\pi \\
0 \\
8 \\
0 \\
\dot{\forall}\end{array}$ & 尊 & 穿 & $\begin{array}{l}\dot{0} \\
\dot{0} \\
\dot{0}\end{array}$ & 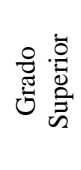 & 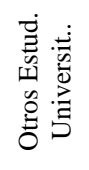 \\
\hline $\begin{array}{c}\mathrm{N}^{\circ} \\
\text { Alumnos/as }\end{array}$ & 130 & 9 & 7 & 13 & 19 & 28 & 19 & 41 & 39 & 49 & 16 & 27 & 3 & 0 & 3 \\
\hline $\begin{array}{c}\% \\
\text { Alumnos/as }\end{array}$ & 93,5 & 6,5 & 5 & 9,4 & 13,7 & 20,1 & 13,7 & 29,5 & 28,1 & 35,3 & 11,5 & 19,4 & 2,2 & 0 & 2,2 \\
\hline
\end{tabular}

Fuente: elaboración propia 
TABLA 4 (A). Sistema de categorías y subcategorías de primer y segundo nivel acordado por los investigadores y empleado para el análisis de los relatos autobiográficos

\begin{tabular}{|c|c|c|}
\hline Categorías & $\begin{array}{l}\text { Subcategorías } \\
\text { nivel } 1\end{array}$ & $\begin{array}{l}\text { Subcategorías } \\
\quad \text { nivel } 2\end{array}$ \\
\hline \multirow{9}{*}{ 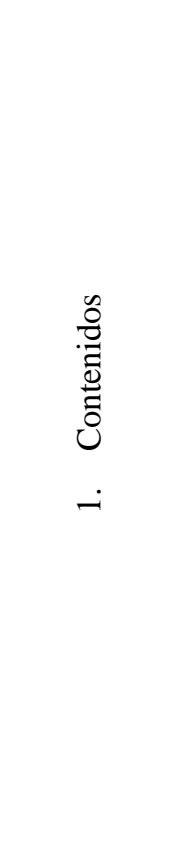 } & \multirow{3}{*}{ Tipología } & Conceptuales \\
\hline & & Procedimentales \\
\hline & & Actitudinales \\
\hline & \multirow{6}{*}{ Поге } & Seres vivos y medio natural \\
\hline & & Cuerpo humano y salud \\
\hline & & Química \\
\hline & & Astronomía \\
\hline & & Física \\
\hline & & Geología \\
\hline \multirow{6}{*}{ 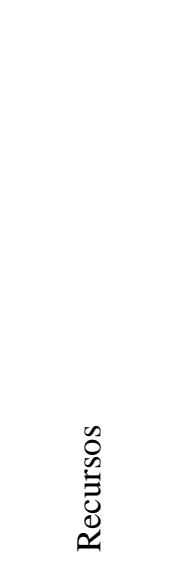 } & \multirow{7}{*}{ Generales } & De expresión corporal (baile, teatro) \\
\hline & & $\begin{array}{l}\text { De expresión plástica (uso de plastilina, } \\
\text { realización de murales o maquetas }\end{array}$ \\
\hline & & Lúdicos (juego y baile) \\
\hline & & Tecnológicos (ordenador) \\
\hline & & Audiovisuales (vídeos, películas) \\
\hline & & Libros de texto \\
\hline \multirow[t]{5}{*}{$\dot{\sim}$} & & Fichas \\
\hline & \multirow{4}{*}{$\begin{array}{l}\text { Característicos de las } \\
\text { ciencias }\end{array}$} & $\begin{array}{l}\text { Salidas a espacios abiertos (parques, bosques, } \\
\text { sierra, reservas, parques naturales) }\end{array}$ \\
\hline & & $\begin{array}{l}\text { Visitas organizadas (granjas-escuela, museos, } \\
\text { parques zoológicos, jardines botánicos) }\end{array}$ \\
\hline & & $\begin{array}{c}\text { Laboratorio y trabajos prácticos (hacer jabón, } \\
\text { plantar árboles, plantar semillas, recoger de } \\
\text { hojas) }\end{array}$ \\
\hline & & Huerto escolar \\
\hline
\end{tabular}

Fuente: elaboración propia 
TABLA 4 (B). Sistema de categorías y subcategorías de primer y segundo nivel acordado por los investigadores y empleado para el análisis de los relatos autobiográficos

\begin{tabular}{|c|c|c|}
\hline Categorías & $\begin{array}{l}\text { Subcategorías } \\
\text { nivel } 1\end{array}$ & $\begin{array}{l}\text { Subcategorías } \\
\text { nivel } 2\end{array}$ \\
\hline \multirow{5}{*}{ 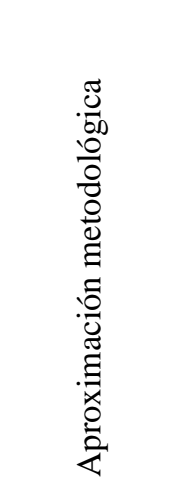 } & \multirow{5}{*}{ Tradicional } & Clases magistrales \\
\hline & & Actividades teóricas \\
\hline & & Trabajo individual \\
\hline & & Aprendizaje memorístico \\
\hline & & Evaluación mediante examen \\
\hline \multirow[t]{2}{*}{-} & \multirow{2}{*}{ Alternativas } & Aprendizaje experiencial \\
\hline & & Trabajo cooperativo \\
\hline \multirow{4}{*}{ 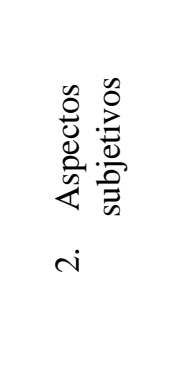 } & $\begin{array}{l}\text { Satisfacción respecto a la } \\
\text { enseñanza recibida }\end{array}$ & \\
\hline & $\begin{array}{l}\text { Opinión sobre el } \\
\text { profesorado }\end{array}$ & \\
\hline & Interés por las ciencias & \\
\hline & $\begin{array}{l}\text { Utilidad de las ciencias en su } \\
\text { vida cotidiana }\end{array}$ & \\
\hline
\end{tabular}

Campamento, museo, casa juventud, etc.

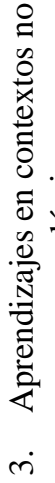

No formales

Voluntario

Monitor de tiempo libre

Con la familia

Con otras compañías

Informales

En los medios de comunicación

Mediante el contacto con la naturaleza

Fuente: elaboración propia 
A continuación, se presentan los resultados obtenidos del análisis de relatos autobiográficos por categorías y subcategorías de análisis, incluyendo: (1) los indicadores cuantitativos (número de relatos en que aparece cierto aspecto respecto al número total de relatos analizados, expresado en porcentaje), (2) y fragmentos de textos literales.

En una vista general de los relatos, casi 2/3 de los participantes afirmó tener recuerdos relacionados con el aprendizaje de las ciencias en la etapa de Educación Infantil, y prácticamente el $90 \%$, tener recuerdos de las etapas educativas posteriores. El 4,7\% restante no mencionó nada sobre dichas etapas, pero tampoco afirmó explícitamente no tener recuerdos. En relación a la narración a lo largo del tiempo, casi $1 / 4$ de los estudiantes (el 23,4\%) mencionaron haber percibido un cambio importante en el paso de Educación Primaria a Educación Secundaria.

\subsection{Contenidos}

En la mayoría de los relatos autobiográficos se hizo referencia a algún tipo de contenido relacionado con las ciencias (83,2\%). De esos contenidos, las referencias más abundantes fueron a los de tipo procedimental (73,4\%), seguidos de los conceptuales $(54,7 \%)$ y los actitudinales (14,5\%). Respecto a los temas concretos que se mencionaron, se consideraron todas las referencias a haber "dado/visto/aprendido" algo, como, por ejemplo: "dimos las plantas", "vimos los animales", “estudiamos el sistema solar”. El 47,7\% de los estudiantes (122 alumnos/as) mencionaron uno o más contenidos. Para una mejor comprensión de los datos, estos temas se agruparon en áreas disciplinares, cuya frecuencia de aparición se presenta en el Gráfico 1, calculada sobre el número total de referencias a temas (que fue de 351).

Los contenidos relativos a las distintas áreas de conocimiento de la Biología, que se han clasificado en las subcategorías "Seres vivos y medio natural" y "Cuerpo humano y salud", fueron los más mencionados por los estudiantes (74\% sobre el total de menciones a temas), y también los que despertaron más su interés, por ejemplo: "Era la asignatura que más me gustaba (Conocimiento del Medio), ya que me interesaba mucho el mundo de las plantas y los animales" (Individuo 140). Por el contrario, los contenidos de Física fueron los menos mencionados (2,6\%) y también los más rechazados, pues cuando los estudiantes los mencionaron, lo hicieron con una carga emocional negativa: "Se me atragantó”, "me aburría”, "no entendía nada”, "me aprobó con la condición de no cogerla en el bachillerato".

GRÁFICO 1. Temas mencionados por los estudiantes, agrupados por áreas disciplinares, junto con su frecuencia de aparición expresada en porcentaje (sobre $\mathrm{N}=351$, número total de menciones)

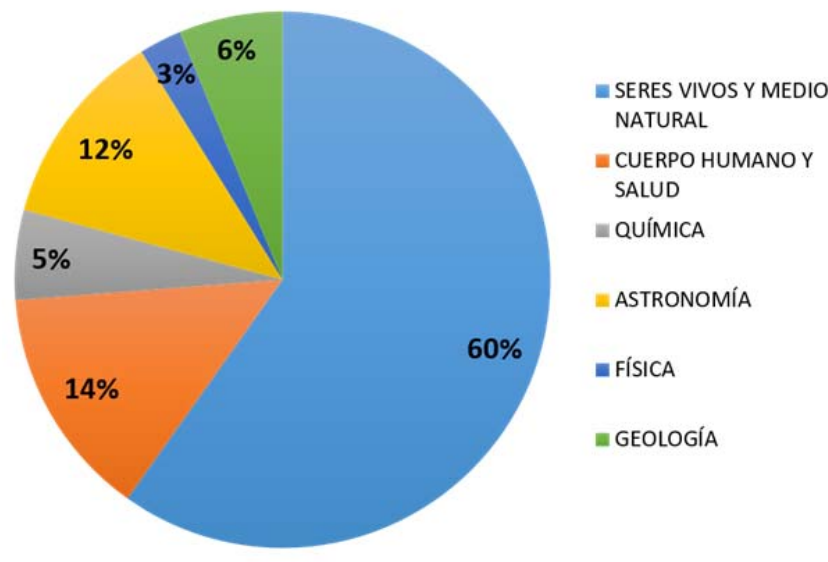

Fuente: elaboración propia 


\subsection{Recursos}

La gran mayoría de estudiantes, 233 en total (el 91\%) mencionó algún recurso didáctico característico de las ciencias, mientras que solo el 30,9\% mencionó algún recurso didáctico de tipo general. Las frecuencias se muestran en el Gráfico 2 y en el Gráfico 3, respectivamente. En relación a los recursos generales, la mitad de los/as alumnos/as (el 49,2\%) hizo referencia a los libros de texto, para indicar o bien que era el único recurso utilizado -y por tanto el eje vertebrador de sus asignaturas de ciencias- o bien que se utilizaba excesivamente, por ejemplo: "Yo de pequeña nunca hice experimentos en mi colegio. Teníamos el libro verde de naturales y punto" (Individuo 5), o "utilizábamos excesivamente el libro, leyéndolo y haciendo un repaso mediante preguntasrespuestas” (Individuo 245).

GRÁFICO 2. Recursos generales mencionados por los/as alumnos/as, con su frecuencia de aparición expresada en porcentaje $(\mathrm{N}=120)$

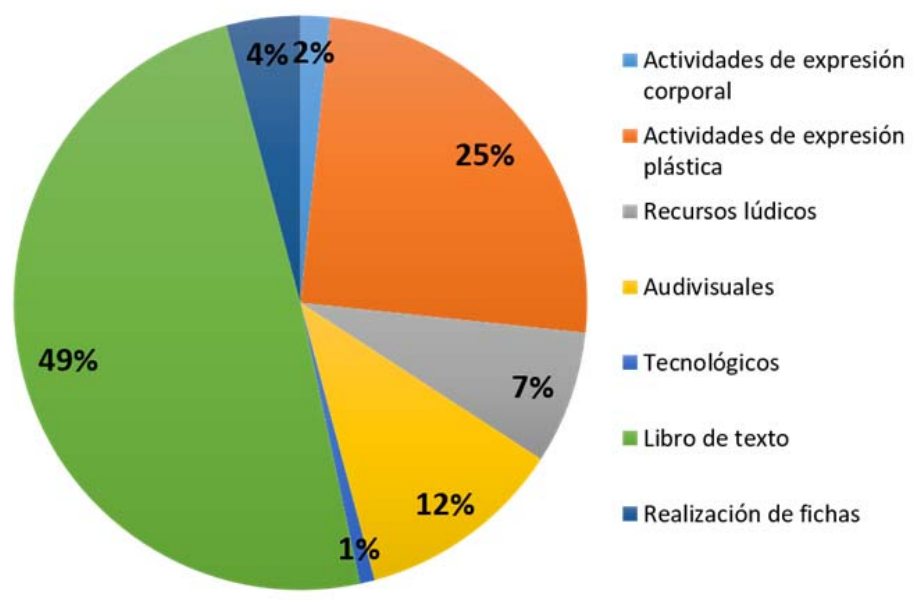

Fuente: elaboración propia

GRÁFICO 3. Recursos característicos de las ciencias mencionados por los/as alumnos/as, con su frecuencia de aparición expresada en porcentaje $(\mathrm{N}=520)$

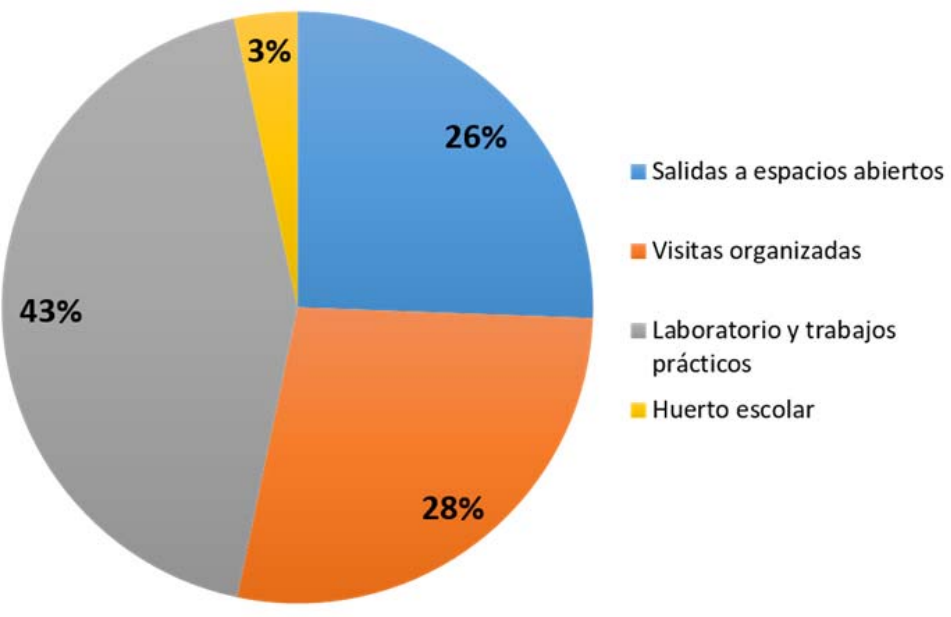

Fuente: elaboración propia 
Respecto a los recursos característicos de las ciencias, destacaron los relatos detallados de salidas a espacios abiertos y las visitas organizadas, a las que se refirieron un total de 133 (25,6\%) y de 144 alumnos/as (27,7\%), respectivamente, por ejemplo: “recuerdo haber ido al Jardín Botánico de Rota, donde nos explicaron cómo plantar, cómo cuidar las plantas, además de ver la diversidad de plantas y animales que en él se encontraba (...) recuerdo haber hecho una excursión al Parque Natural de Doñana” (Individuo 4), "Realizamos alguna que otra excursión al zoológico, pinar, etc.” (Individuo 36), o "En la primaria he realizado diferentes actividades relacionadas con la ciencia como salidas al campo, diferentes excursiones como el zoo de Jerez, o selwo marina, y también la visita al castillo de las Guardas en Sevilla” (Individuo 52). Los/as alumnos/as también recordaron los trabajos prácticos en el laboratorio o en otros espacios, tales como cuidar plantas, secar hojas de árboles, llevar animales al aula y cuidarlos, coleccionar piedras, hacer un herbario, hacer jabón etc., a los que se refirieron un total de 225 alumnos/as (43,3\%), siempre de forma neutra o positiva, por ejemplo: "En segundo de primaria, con un vaso de plástico, algodón, una legumbre, y regándola, hacíamos nuestra propia planta, era muy divertido y todos los días lo mirábamos entusiasmados para ver si había crecido" (Individuo 242). Las actividades prácticas aparecieron más asociadas a las etapas de Infantil y Primaria, mientras que el laboratorio apareció en Secundaria.

El trabajo en el laboratorio se describió como puntual y no habitual. Además, no siempre fue para experimentar o indagar, sino que en ocasiones se acudía allí para presenciar las demostraciones del docente, como expresa este estudiante: "Un día, fuimos al laboratorio en el que únicamente cogimos apuntes de un experimento que el profesor realizó" (Individuo 150), o "lo único que hacíamos en el laboratorio era dar clases teóricas” (Individuo 232). Los estudiantes recuerdan haber utilizado el microscopio, haber realizado disecciones de órganos de animales (vaca, cerdo), etc. La disección de órganos es recordada con una carga emocional negativa por cierto número de estudiantes: "teníamos que diseccionar un corazón, cosa que no me gustaba nada y te obligaban a realizar” (Individuo 225), o “diseccioné por primera vez unos pulmones, me fue muy desagradable y me costó mucho” (Individuo 228).

Finalmente, las referencias al uso de huerto escolar fueron más bien escasas: un total de 18 (3,5\%). Un estudiante señaló: "En esta etapa (secundaria) en el colegio hicimos un huerto donde cada uno se encargaba de un tipo de alimento (sembrar, regar, etc.)” (Individuo 130), otro: “...realicé muchas actividades en el huerto del colegio con mis compañeros/as como por ejemplo, regar las plantas, cultivar alimentos, etc.” (Individuo 92), y un tercero: "Otra de las actividades que realizamos con el colegio fue un ecohuerto. Plantábamos para luego recoger sus frutas como tomates, patatas, etc.” (Individuo 25).

\subsection{Aproximaciones metodológicas}

Los aspectos metodológicos tuvieron un papel menos relevante en los relatos de los/as alumnos/as, puesto que más de la mitad de ellos no hicieron referencia alguna a los mismos. Se encontraron 114 relatos (44,5\%) en que los estudiantes referían haber vivenciado una aproximación metodológica tradicional de la enseñanza de las ciencias, frente a $8(3,1 \%)$ en que hubo alusiones a aproximaciones alternativas, que aparecieron solo en los/as alumnos/as de la Facultad de Educación de Soria.

En concreto, el 8,2\% de estudiantes se refirió a las clases magistrales: "La mayoría de las clases eran magistrales, los profesores nos daban los contenidos y después realizábamos sus respectivas actividades, siempre basándose en los libros de texto" (Individuo 199); el 25\%, a que se les requería la realización de actividades teóricas: "En esta etapa (Secundaria) no recuerdo ninguna excursión ni viajes, sólo dábamos la teoría y hacíamos los exámenes” (Individuo 36), "Las clases eran totalmente teóricas y el docente explicaba los contenidos que aparecían en el libro de texto" (Individuo 170), y el 14,1\%, al aprendizaje como memorización de contenidos: “Aprobaba pero sin entender ni aprender nada porque memorizaba todos los contenidos” (Individuo 59), “...puedo decir 
que aprendí ciencias, más bien lo memoricé...” (Individuo 78), "Era una asignatura que me la estudiaba de memoria y casi sin entender” (Individuo 81). El uso de esta aproximación metodológica se acentuó con el paso de Primaria a Secundaria, según narraron los estudiantes: “en la etapa de secundaria, todas las clases de ciencias se volvieron mucho más teóricas, subrayando el libro, haciendo sus actividades de forma individual y realizando un examen de desarrollo escrito" (Individuo 160), con connotaciones negativas en lo que se refiere a motivación por las ciencias: “(En Primaria) No me suponía ningún esfuerzo asimilar la información, es más, adoraba conocer los planetas que componían el sistema solar o el funcionamiento de un volcán...(En Secundaria) a partir de entonces dejó de gustarme” (Individuo 190), o “ En la etapa de secundaria esta asignatura pasó a ser un suplicio (...) decidí que las ciencias no era el camino que iba a elegir y me decidí por las ciencias sociales" (Individuo 180). Algunos estudiantes señalaron que "de manera muy práctica (en etapas anteriores) se interiorizaban mucho mejor los contenidos” (Individuo 228).

Un aspecto destacable es el número de alusiones que se hizo a la evaluación mediante examen, un total de 19; más teniendo en cuenta que no hubo referencia alguna a otro modo o instrumento de evaluación: "se limitaban a leer lo que ponía en los libros y simplemente nos preparaban para realizar los exámenes parciales a lo largo del curso” (Individuo 252), “convertirnos en meros espectadores que escuchábamos las explicaciones que luego deberíamos repetir en el examen” (Individuo 225). De este modo, el esquema más usual de aprendizaje que los estudiantes relataron fue: memorización del concepto, práctica y reproducción.

Una minoría de alumnos/as mencionó haber vivenciado un aprendizaje experiencial (0,8\%), y haber tenido experiencias de aprendizaje colaborativo (2\%), mayoritariamente en la etapas de Infantil y, en menor grado, en la de Primaria, por ejemplo: “...en el recreo manipulábamos con algún tipo de planta, con la nieve...” (Individuo 230) y “...cada día uno de la clase les tenía que poner de comer y cambiarles los periódicos, era un trabajo en el que todos participábamos (...) eran experiencias en las que manipulábamos y unos a otros nos ayudábamos” (Individuo 225). Este tipo de aprendizaje se considera motivador: "De las ciencias naturales la verdad tengo buenos recuerdos porque la asignatura era divertida y vivíamos muchas experiencias” (Individuo 100).

\subsection{Aspectos subjetivos}

Respecto a aspectos subjetivos, solo un 18,8\% de los estudiantes dijo estar satisfecho en relación a la enseñanza de las ciencias recibida en su trayectoria académica anterior al ingreso en la universidad, mientras que otro 20,3\% dijo no estarlo; el resto (60,9\%) no se manifestó claramente al respecto. Los estudiantes hicieron afirmaciones del tipo: "Mi experiencia en ciencias hubiera sido mejor si el profesor hubiera utilizado otros instrumentos para la enseñanza de la materia" (Individuo 150). Del total de estudiantes, el 39,5\% expresó algún tipo de opinión sobre el profesorado de las disciplinas de ciencias; ésta opinión fue positiva en un $28,1 \%$ y negativa en un 19,9\%. Así, en sus relatos los/as alumnos/as subrayaron la figura del docente en un sentido u otro: para descartar la asignatura y las opciones futuras relacionadas con ella: “... el profesor hacía la asignatura muy fea y no disfrutábamos dando esa asignatura. Era muy difícil y por ello me decidí por las ciencias sociales..." (Individuo 71), "El docente que tuve me influyó muy negativamente y desde ese momento supe que no escogería el camino de las ciencias.” (Individuo 81), o para decantarse por continuarla en Bachillerato: “...el profesor dejó una gran huella en mí, la cual hizo que eligiera el bachillerato de ciencias.” (Individuo 82), “El profesor que nos daba clases era muy bueno. Este profesor hizo que me gustara mucho la Física y Química” (Individuo 152). A menudo vincularon la figura del docente con aspectos metodológicos: si el docente era activo y le gustaba la materia, hacía una clase más participativa, y organizaba más salidas y actividades prácticas, aunque no restaba importancia a la memorización de contenidos. Por ejemplo: “...tengo también un grato recuerdo de mis profesores ya que, aunque nos obligaron a aprender de "pe" a "pa" los elementos de la famosa tabla...aprendí a usar la ciencia y la utilidad que esta tenía para nuestras vidas” 
(Individuo 5), "recuerdo que mi profesor nos llevaba al campo a experimentar....llegaba a casa muy alegre y entusiasmada, y se lo contaba a mi padre y a mi hermano" (Individuo 241), o "El profesor que nos impartía esta asignatura hacía que aprendiéramos de una forma divertida, con mucho ejemplo práctico y muchas excursiones” (Individuo 243).

Además, el 39,1\% de los estudiantes afirmaron estar actualmente interesados en las ciencias, con comentarios del tipo "me interesan mucho las ciencias" o "siempre me han interesado las ciencias". Es interesante señalar que tan solo el 5,5\% de los estudiantes (14 en total) dijo haber encontrado utilidad en los aprendizajes de ciencias, justificándolo: “...gracias a la ciencia puedo comprender el motivo de nuestra existencia” (Individuo 8) o "Siempre me gustaron las ciencias... tal vez porque siempre lo viví como algo interesante que se podía aplicar al día a día” (Individuo 46). Un escaso 1,2\% (3 estudiantes) explicitó que sus aprendizajes no le habían resultado útiles para su vida cotidiana, y el resto (93,3\%) no hizo referencia explícita a este aspecto.

\subsection{Aprendizajes en contextos no académicos}

Respecto a las experiencias de aprendizaje de las ciencias vividas en contextos no académicos, el 26,2\% de los estudiantes dijo haber aprendido de modo no formal, en campamentos (21,5\%) o ejerciendo como monitor de tiempo libre (2,7\%) o voluntario (2\%). A nivel de aprendizajes informales, el 32\% de los estudiantes afirmó haber aprendido ciencias junto con su familia, por ejemplo: "fui con mi familia a un museo", "tengo contacto actualmente con las ciencias porque voy con mi familia al campo", "mis padres me explicaban cosas de ciencia”, "mi padre/madre era aficionado a la botánica/canarios/biología” o "siempre iba al campo de mi abuelo". Un 9,8\% afirmó haber aprendido ciencias a través del propio contacto con la naturaleza. Un 7\% dijo haber realizado actividades relacionadas con las ciencias con amigos u otras compañías; y otro 7\%, haber aprendido ciencias mediante series de televisión, documentales, revistas, etc. (medios de comunicación).

Respecto a estos aprendizajes fuera de las instituciones educativas, se describieron experiencias con familiares o amistades, en asociaciones, mediante actividades de ocio y tiempo libre, etc., por ejemplo: “...me gustaría indicar la cantidad de cosas y actividades que podrían trabajar los niños en la escuela con esta asignatura y que yo, desgraciadamente, no pude en la escuela, pero sí con mi familia” (Individuo 3) o "Recuerdo un día que salimos al campo de excursión con mi familia donde vi una especie de escarabajo muy raro y no sabía su nombre. Cuando llegué a casa me puse a buscar que clase era” (Individuo 16). Los estudiantes diferenciaron los aprendizajes académicos de los que se producen en otros entornos, que a menudo no concibieron propiamente como aprendizaje, por ejemplo: "Yo he sido una persona que estado en contacto siempre con la naturaleza, las cosas que me han llamado la atención las he investigado, y sí que me han gustado, pero yo creo que es más por iniciativa propia que por obligación de aprenderlo como pasaba en el colegio” (Individuo 19), o:

Fuera de la escuela no me he interesado por estudiar nada relacionado con las ciencias, quizá porque me parecía algo bastante complicado con alguien que te guiaba como para ponerme por mí misma a estudiar o intentar aprender algo de ciencias. Eso sí, he de decir que los típicos experimentos caseros muy sencillos que salen en la tele siempre me ha gustado hacerlos bien con mi hermano en casa o con mis amigas (Individuo 17).

\section{DisCUSIÓN Y CONCLUSIONES}

En el presente estudio se ha empleado como técnica de recogida de datos la narración autobiográfica, focalizada en las experiencias de enseñanza-aprendizaje de las ciencias previas al 
ingreso en la universidad de los/as maestros/as en formación inicial. Esta técnica, que se inscribe dentro de un enfoque de investigación cualitativo, ha mostrado ser útil en relación a los objetivos de esta investigación, pues nos ha permitido escuchar las voces de nuestros estudiantes cuando relatan cómo les enseñaron ciencias, qué aprendieron, cómo se sintieron, qué les motivaba, qué les resultaba difícil, etc. Además, mediante un recuento de frecuencias - conteo del número de ocasiones en que cierta información se repite-, esta técnica nos ha permitido tener una aproximación cuantitativa a los fenómenos estudiados, y evidenciar sobre qué hablan más nuestros estudiantes, y, por tanto, a qué temas conceden mayor importancia.

A nivel didáctico, la técnica empleada posee también un valor importante, y es que cada una de las categorías analizadas puede resultar útil como punto de partida para reflexionar conjuntamente con nuestro alumnado y proponer soluciones y alternativas para la mejora de la enseñanza-aprendizaje de las ciencias. Es decir, que las historias de vida, al igual que otros elementos, denominados por Astudillo, Rivarosa y Ortiz (2014) dispositivos de formación secuencias didácticas, diarios de trabajo o grupos de discusión-, suponen una oportunidad para movilizar la reflexión consciente sobre determinados aspectos implicados en el conocimiento práctico del profesorado. Así, esta técnica se convierte a la vez en un recurso didáctico, que puede contribuir a formar un profesorado más crítico, más consciente de cómo les han sido enseñadas las ciencias a lo largo de su etapa escolar.

En los relatos analizados se observaron escenarios semejantes a los detectados en estudios anteriores, por ejemplo: la enseñanza entendida como una estructura de tareas, el aprendizaje como la reproducción de contenidos del currículum, la escuela como único espacio de aprendizaje, o la idea de que sólo es válido aquello que se evalúa. En la mayoría de relatos apareció un modelo de enseñanza-aprendizaje transmisor que reproduce una visión fragmentaria, acumulativa y absoluta de las ciencias en la que están ausentes los aspectos éticos y de compromiso social; que separa los contenidos de la metodología y de la evaluación; y que no considera la dimensión colectiva del aprendizaje (Rivas y Leite, 2013). Por tanto, los estudiantes en su gran mayoría reflejaron una enseñanza de las ciencias con finalidad exclusivamente propedéutica y centrada en los conceptos.

Emerge también que nuestros estudiantes consideran propiamente aprendizaje tan solo el aprendizaje formal, mientras que el aprendizaje en contextos no académicos -tanto informal como no formal (Carrasco, Jadue, Letelier y Oliva, 2012)-, no lo es, a pesar de que los conocimientos que se pueden adquirir de manera informal parecen ser útiles desde la perspectiva de la enseñanza de las ciencias en el aula, según Oliva, Matos, Bueno, Bonat, Domínguez, Vázquez y Acevedo (2004), entre otros. Así, las narraciones sobre el aprendizaje de las ciencias durante la etapa de Infantil se asemejan a las que se hacen en relación a contextos no académicos: destacan aspectos como la emoción o el interés. La narración cambia en relación a la etapa de Primaria, y este cambio se agudiza con el paso a Secundaria: a partir de entonces, se asocia aprender ciencias con usar el libro de texto y con un temario organizado y cerrado que hay que memorizar y reproducir en los exámenes, que constituyen el único medio de evaluación.

Nuestros resultados muestran además que existen disciplinas mejor valoradas que otras; así, los contenidos de Biología no sólo fueron los más mencionados, sino también los que más interés suscitaron. Estos resultados coinciden con investigaciones realizadas sobre alumnos/as de Secundaria, quienes consideran más difíciles y aburridas Física y Química que Biología y Geología (Solbes, Montserrat y Furió, 2007; Méndez, 2015). En estudios realizados en futuros profesores de Secundaria se han obtenido resultados similares; éstos manifiestan emociones más positivas respecto a los contenidos de Biología cuando recuerdan sus experiencias como alumnos/as de Secundaria (Costillo, Borrachero, Brígido y Mellado, 2013). Similarmente, en futuros docentes de Primaria, Brígido, Bermejo, Conde, Borrachero y Mellado (2010) obtuvieron que los contenidos de Ciencias Naturales despertaban en los estudiantes emociones positivas, mientras que los de Física y Química se asociaban más a emociones negativas. 
Otro aspecto a destacar en relación a los contenidos es que no se alude en los relatos a aspectos referentes a la Naturaleza de la Ciencia (NdC), tan relevantes como el papel de la mujer en la ciencia, la construcción de la ciencia, o el propio proceso científico, acordes con el enfoque que propone el movimiento Ciencia-Tecnología-Sociedad (CTS), que ha venido promoviendo en las últimas décadas una enseñanza de las ciencias más significativa y contextualizada, estrechamente vinculada con la vida cotidiana del alumnado, de la que existen valiosos ejemplos (Girón, Blanco y Lupión, 2015), y que defiende la alfabetización científico-tecnológica (ACT) para todas las personas (Vázquez-Alonso, Acevedo-Díaz y Manassero-Mas, 2015). Emerge nuevamente la visión puramente propedéutica de la enseñanza de las ciencias imperante en nuestras escuelas.

Respecto al uso de recursos, algunos estudios revelan la existencia de una correspondencia con el grado de interés que se despierta en el alumnado. De Orta, Reyes y De las Heras (2016) indican que las salidas al medio despiertan en los/as alumnos/as más emociones positivas que negativas, lo cual parece aumentar su motivación y predisponerles al aprendizaje. Lo mismo ocurre con otros recursos característicos de la enseñanza de las ciencias, como los trabajos prácticos, uno de cuyos objetivos es motivar a los estudiantes (Caamaño, 2003). En las narraciones que hemos analizado, los/as futuros maestros/as recuerdan particularmente esas experiencias. Por otra parte, y en una secuencia temporal, se observa que los trabajos prácticos son más frecuentes en Infantil y Primaria, mientras que en Secundaria se acude al laboratorio pero con fines demostrativos, más semejantes a experiencias de cátedra que para plantear pequeñas investigaciones. Probablemente aquí residen los motivos por los que en las aulas de Infantil están poco presentes las metodologías propias de las ciencias: los/as maestros/as se consideran insuficientemente formados (Cantó, de Pro y Solbes, 2016).

En conclusión, se evidencia la necesidad de promover, desde la formación inicial de maestros/as, relatos con experiencias distintas de aprendizaje, que incluyan la emocionalidad y el deseo de pensar, relacionar, valorar, descubrir e intervenir en el mundo. Visto que los aprendizajes en contextos no académicos se revelan como muy importantes en la trayectoria vital de nuestros estudiantes, es necesario visibilizarlos y abrir una reflexión sobre ellos, poniéndolos en diálogo con el conocimiento escolar sobre ciencias, para aprovechar las dimensiones emocionales, de actitudes y valores que conllevan, habitualmente muy positivas. En ambos sentidos parece aconsejable que los docentes universitarios exploremos metodologías activas y participativas, consideremos el uso de contextos de aprendizaje alternativos al aula, como los huertos ecodidácticos (Eugenio y Aragón, 2016), y otras estrategias de aprendizaje, como los modelos analógicos (Jiménez-Tenorio, Aragón y Oliva, 2016).

\section{Referencias}

Astudillo, C., Rivarosa, A., y Ortiz, F. (2014). Reflexión docente y diseño de secuencias didácticas en un contexto de formación de futuros profesores de ciencias naturales. Perspectiva Educacional. Formación de Profesores, 53(1), 130-144.

Bisquerra, R., y Pérez, N. (2012). Educación emocional: estrategias para su puesta en práctica. Avances en Supervisión Educativa, 16, 1-11.

Brígido, M., Bermejo, M.L., Conde, C., Borrachero, A.B., y Mellado, V. (2010). Estudio longitudinal de las emociones en ciencias de estudiantes de maestro. Revista GalegoPortuguesa de Psicoloxía e Educación, 18(2), 161-179.

Bryan, L.A., y Abell, S.K. (1999). The development of professional knowledge in learning to teach elementary science. Journal of Research in Science Teaching, 36, 121-139.

Caamaño, A. (2003). Los trabajos prácticos en ciencias. En M.P. Jiménez (coord.), Enseñar ciencias (pp. 95-118). Barcelona: Graó 
Cantó, J., de Pro, A., y Solbes, J. (2016) ¿Qué ciencias se enseñan y cómo se hace en las aulas de educación infantil? La visión de los maestros en formación inicial. Enseñanza de las Ciencias, 34(3), 25-50.

Carrasco, R., Jadue, F., Letelier, M., y Oliva, C. (2012). Estudio exploratorio sobre aprendizaje noformal e informal en estudiantes y egresados universitarios. Calidad en la Educación, 36, 149-184.

Carniatto, I., y Fossa, A.M. (1998). La creencia docente y los obstáculos epistemológicos: una investigación en la enseñanza con profesores del curso de ciencias biológicas. En E. Banet y A.J. de Pro (Eds.), Investigación e innovación en la enseñanza de las ciencias (pp. 196-203). Murcia: DM.

Costillo, E., Borrachero, A.B., Brígido, $\mathrm{M}^{\mathrm{a}}$., y Mellado, V. (2013). Las emociones sobre la enseñanza-aprendizaje de las ciencias y las matemáticas de futuros profesores de Secundaria. Revista Eureka sobre Enseñanza y Divulgación de las Ciencias, 10 (Núm. Extraordinario), 514-532.

Delval, J. (2002). El juego. México: siglo XXI.

De Orta Á., Reyes, R., y De las Heras, $M^{a}$.Á. (2016). Repercusiones de la forma de enseñar: emociones que despiertan diferentes tipos de actividades. Campo Abierto, 35(1), 189-204.

Eugenio, M., y Aragón, L. (2016). Experiencias en torno al huerto ecológico como recurso didáctico y contexto de aprendizaje en la formación inicial de maestros en infantil. Revista Eureka sobre Enseñanza y Divulgación de las Ciencias, 13(3), 667-679.

Ferreyra, A. (2012). Creencias y concepciones docentes sobre la evaluación de los aprendizajes en el contexto universitario (Tesis de Licenciatura). Pontificia Universidad Católica del Perú, Lima-Perú.

Furió, C., y Carnicer, J. (2002). El desarrollo profesional del profesor de ciencias mediante tutorías de grupos cooperativos. Estudio de ocho casos. Enseñanza de las Ciencias, 20(1), 47-73.

Garritz, A. (2010). Pedagogical Content Knowledge and the affective domain of Scholarship of Teaching and Learning. International Journal for the Scholarship of Teaching and Learning, 4(2), 1-6.

Garritz, A., y Ortega-Villar, N.A. (2013). El aspecto afectivo en la enseñanza universitaria. Cómo cinco profesores enseñan el enlace químico en la materia condensada. En V. Mellado, L.J. Blanco, A.B. Borrachero y J.A. Cárdenas (Eds.), Las Emociones en la Enseñanza y el Aprendizaje de las Ciencias y las Matemáticas (pp. 279-306). Badajoz: DEPROFE.

Gil-Pérez, D. (1991). ¿Qué hemos de saber y saber hacer los profesores de ciencias? Enseñanza de las Ciencias, 9(1), 69-77.

Girón, J.R., Blanco, A., y Lupión, T. (2015). Uso de la publicidad de un producto alimenticio para aprender un modelo sobre las defensas en el intestino humano. Un estudio en $3^{\circ}$ de ESO. Revista Eureka sobre Enseñanza y Divulgación de las Ciencias, 12(2), 278-293.

Hernández, R., Fernández, C., y Baptista, M. (2014). Metodología de la investigación. México D.F.: McGraw-Hill Interamericana.

Jiménez-Tenorio, N., Aragón, L., y Oliva, J.M. (2016). Percepciones de estudiantes para maestros de educación primaria sobre los modelos analógicos como recurso didáctico. Enseñanza de las ciencias, 34(3), 91-112.

Lederman, N.G. (1992). Student's and teacher's conceptions of nature of science: A review of the Research. Journal of Research in Science Teaching, 29(4), 331-359.

Mellado, V. (1996). Concepciones prácticas de aula de profesores de ciencias, en formación inicial de primaria y secundaria. Enseñanza de las Ciencias, 14(3), 289-302.

Mellado, V. (2001). ¿Por qué a los profesores de ciencias nos cuesta tanto cambiar nuestras concepciones y modelos didácticos. Revista Interuniversitaria de Formación del Profesorado, 40, $17-30$. 
Mellado, V. (2003). Cambio didáctico del profesorado de ciencias experimentales y filosofía de la ciencia. Enseñanza de las Ciencias, 21(3), 343-358.

Mellado, V., Borrachero, A.B., Brígido, M., Melo, L.V., Dávila, M.A., Cañada, F., Conde, M.C, Costillo, E., Cubero, J., Esteban, R., Martínez, G., Ruiz, C., y Sánchez, J. (2014). Las emociones en la enseñanza de las ciencias. Enseñanza de las Ciencias, 32(3), 11-36.

Méndez, D. (2015). Estudio de las motivaciones de los estudiantes de secundaria de física y química y la influencia de las metodologías de enseñanza en su interés. Educación $X X 1$, 18(2), 215-235.

Miles, M.B., Huberman, A.M., y Saldaña, J. (2014). Qualitative data analysis: A methods sourcebook. Thousand Oaks: Sage.

Muñoz, M., y Bisquerra, R. (2013). Diseño, aplicación y evaluación de un plan de educación emocional en Guipúzcoa: análisis cuantitativo. EduPsykhé, 12(1), 3-21.

Olitsky, S., y Milne, C. (2012). Understanding engagement in science education: The psychological and the social. En B.J. Fraser, K.G. Tobin y C.J. McRobbie (Eds.), Second International Handbook of Science Education (pp. 9-33). Dordrecht: Springer.

Oliva, J.M., Matos, J., Bueno, E., Bonat, M., Domínguez, J., Vázquez, A., y Acevedo, J.A. (2004). Las exposiciones científicas escolares y su contribución en el ámbito afectivo de los alumnos participantes. Enseñanza de las Ciencias, 22(3), 425-440.

Porlán, R., Rivero, A., y Martín, R. (1997). Conocimiento profesional y epistemología de los profesores I: teoría métodos e instrumentos. Enseñanza de las Ciencias, 15(2), 155-171.

Porlán, R., y Rivero, A. (1998). El conocimiento de los profesores. Sevilla: Díada.

Porlán, R., Rivero, A., y Martín, R. (1998). Conocimiento profesional y epistemología de los profesores II: estudios empíricos y conclusiones. Enseñanza de las Ciencias, 16(2), 271-288.

Pontes, A., Serrano, R., y Poyato, F.J. (2012). Concepciones y motivaciones sobre el desarrollo profesional docente en la formación inicial del profesorado de educación secundaria. Revista Eureka sobre Enseñanza y Divulgación de las Ciencias, 10, 533-551.

Rivas, J.I., y Leite, A. (2013). Aprender la profesión desde el pupitre. Cuadernos de Pedagogía, 436, 34-37.

Ruiz, C., Da Silva, C., Porlán, R., y Mellado, V. (2005). Construcción de mapas cognitivos a partir del cuestionario INPECIP. Aplicación al estudio de la evolución de las concepciones de una profesora de secundaria entre 1993 y 2002. Revista Electrónica de la Enseñanza de las Ciencias, 4(1), 1-21.

Sanmartí, N. (2001). Enseñar a enseñar Ciencias en Secundaria: un reto muy complicado. Revista Interuniversitaria de Formación del Profesorado, 40, 31-48.

Savin-Baden, M., y Major, C. H. (2013). Qualitative research: The essential guide to theory and practice. Nueva York: Routledge.

Solbes, J., Montserrat, R., y Furió, C. (2007). El desinterés del alumnado hacia el aprendizaje de la ciencia: implicaciones en su enseñanza. Didáctica de las Ciencias Experimentales y Sociales, 21, 91-117

Sparkes, A., y Smith, B. (2014). Qualitative research methods in sport, exercise and health: From process to product. Nueva York: Routledge.

Tedesco, J. (2006). Prioridad a la enseñanza de las ciencias: una decisión política. Recuperado de https://www.oei.es/historico/cienciayuniversidad/spip.php?article3558 $\quad$ [10 de febrero de 2019]

Thagard, P. (2008). Conceptual change in the history of science: Life, mind, and disease. En: S. Vosniadou (Ed.), International handbook of research on conceptual change (pp. 374-387). London: Routledge.

Tobin, K. (1993). Referents for making sense of science teaching. International Journal of Science Education, 15(3), 241-254. 
Tobin, K.G., Tippins, D.J., y Gallard, A.J. (1994) Research on Instructional Strategies for Teaching Science. En D. L. Gabel (Ed.), Handbook of Research on Science Teaching and Learning (pp. 45-93). New York: National Science Teachers Association.

Tójar, J.C. (2006). Investigación cualitativa. Comprender y actuar. Madrid: La Muralla.

Vázquez, A., y Manassero, M.A. (2007). En defensa de las actitudes y emociones en la educación científica (I): evidencias y argumentos generales. Revista Eureka sobre Enseñanza y Divulgación de las Ciencias, 4(2), 247-271.

Vázquez-Alonso, Á., y Manassero-Mas, M.A. (2015). Hacia una formación inicial del profesorado de ciencias basada en la investigación. Revista Española de Pedagogía, 261, 343-363.

Vázquez-Alonso, Á., Acevedo-Díaz, J.A., y Manassero-Mas, M.A. (2005). Más allá de la enseñanza de las ciencias para científicos: hacia una educación científica humanística. Revista Electrónica de Enseñanza de las Ciencias, 4(2).

\section{Cómo CITAR ESTE ARTÍ́CULO}

Eugenio-Gozalbo, M., Aragón Núñez, L., Vicente Martorell, J. J. y Jiménez-Tenorio, N. (2019). Análisis de las experiencias de aprendizaje de las ciencias de los/as maestros/as de Educación Infantil y Primaria en formación inicial a través de sus relatos de vida. Didáctica de las ciencias experimentales y sociales, 37, 107-126. DOI: 10.7203/DCES.37.14303 
